\title{
Enhancing Transparency and Openness at the Journal of Research in Personality
}

\author{
Richard E. Lucas ${ }^{\mathrm{a}}$, M. Brent Donnellan ${ }^{\mathrm{b}}$ \\ ${ }^{a}$ Department of Psychology, Michigan State University \\ ${ }^{b}$ Department of Psychology, Texas AEM University
}

Keywords: Transparency, Openness, Replications, Reproducibility, Power, Methods

Transparency and openness are critical features of scientific investigations. These features allow independent researchers to evaluate claims made within journal articles, they allow for a deeper understanding of the phenomena being investigated, and ideally, they improve the reproducibility of scientific research. As many have noted (e.g., Nosek et al., 2015), however, current incentive structures do not always reward individual researchers who prioritize openness and transparency, even if such practices would increase the credibility and quality of the research. Thus, improving scientific practices requires additional efforts that focus on changing the existing incentive structure.

Four years ago, the editors of this journal instituted a set of policies designed to address concerns about transparency, openness, and reproducibility (Lucas \& Donnellan, 2013). As part of that effort, we encouraged submission of replication studies, we required authors to provide an explicit discussion of sample size decisions and power, and we asked authors explicitly about data availability and sharing. Since that time, we have also instituted "badges" for open and transparent practices, including badges for open data, open materials, and preregistration (Kidwell et al., 2016). Although we believe that these policy changes have been successful, we also anticipated that revisions and updates would be needed as innovations in the field emerged and norms evolved.

The Journal of Research in Personality has already endorsed the Transparency and Openness Promotion [TOP] guidelines (Nosek et al., 2015), and we are now ready to announce our position on each of the guidelines listed. These guidelines go beyond a statement of philosophy; they also have implications for journal policy. Indeed, as part of this announcement, we will introduce new submission options at the journal and new requirements for papers being considered here. We believe that these changes are good for science and will improve the quality of research that is published in the journal.

Below we describe in detail the "level" that the Journal of Research in Personality has selected for each of the TOP guidelines. However, to provide a broad summary, we note the following policies and policy changes. Authors are encouraged to attend to these issues before submission to avoid any delays in processing their manuscripts. More detailed instructions will soon be published on the journal's on-line guide to authors.

Email addresses: lucasri@msu.edu (Richard E. Lucas), mbdonnellan@tamu.edu (M. Brent Donnellan)
- Sample Size and Power: The journal will continue to enforce our previously instituted policies regarding the inclusion of a statement describing how sample sizes were determined and how the sample size affects power and precision.

- Open Data, Code, and Materials: The default for all submitted manuscripts will be that data, code, and materials will be made public for other researchers to access; though certain exceptions will be allowed as long as they are stated explicitly at submission and in a note in the published article.

- Preregistration: The journal encourages (but does not require) preregistration and will ask authors to provide a link to the preregistration to reviewers for verification.

- Registered Reports: The journal will adopt the optional use of registered reports (Nosek \& Lakens, 2014).

- Replication Studies: The journal will continue to encourage submission of replication studies, especially studies that attempt to replicate work published in our pages. We will also adopt new policies for the evaluation of replication studies, including the use of registered reports when possible, and results-blind review for replication studies that have already been conducted.

- Author Contributions: Papers will be required to list the specific contribution that each author made to the published paper.

- Statements on Constraints on Generalizability: To encourage appropriate conclusions and inform future replication attempts, authors will be asked to discuss whether they expect any constraints on the generalizability of their findings to other contexts, populations, etc (see, Simons et al., 2017).

\section{Sample Size and Power}

In our previous editorial, we adopted a policy requiring authors to discuss sample size decisions and the effects of these decisions on statistical power and precision. This discussion of sample size is expected in all manuscripts, and the power of the 
study is a factor that editors and reviewers use when evaluating the conclusions that the authors draw in their work. Because there has been some confusion about what is required for JRP submission, we will take this opportunity to clarify expectations.

When describing their sample, authors should state how sample sizes were determined. If sample sizes were not based on a formal power analysis (e.g., if sample sizes were determined by convenience, resource limitations, or the use of archival data), authors should state this explicitly. Regardless of how sample size decisions were made, authors should discuss the anticipated power of their study for detecting effects that might reasonably be expected. If no prior work is available to inform effect size expectations, then authors should assume that effects will be close to those that are typical for the field (e.g., $r=.20$; Gignac \& Szodorai, 2016; Richard et al., 2003). If prior work can be used to inform expectations, then authors should discuss this empirical work. Briefly reporting representative effect sizes (and sample sizes) is encouraged. If necessary, authors can provide more extensive details about previous studies in supplementary materials.

Authors should be realistic in their assessment of prior work and not just rely on the largest effects that have been reported. In addition, published effects are likely overestimates of the true effect given publication bias, and this should be taken into account. Authors should also consider whether there are design differences from previous work that would lead to different expectations for the current study. For instance, if prior work has focused on main effects, and the current study tests an interaction, then expectations about effect sizes should be adjusted based on the change in hypothesis and design. In some cases, there might not be any precedent for particular interactions. Realistic expectations about the form of the interaction and simulation studies might be required to inform readers about power in these cases. Extremely large sample sizes are often required to detect small interactions with measured variables and the typical levels of measurement error found in personality psychology (i.e., sample sizes over 1,000; Cohen et al., 2003; Simonsohn, 2014). After considering the above issues, authors should describe the power of their study to detect their expected effects. Moving forward, we strongly encourage JRP authors to adopt sample size planning techniques that increase the accuracy of parameter estimation (e.g., Maxwell et al., 2008).

The editors recognize that many of the steps in the process described above rely on subjective judgments. One of our objectives is to make these judgments explicit so editors, reviewers, and readers can weigh them appropriately when evaluating the research. We also acknowledge that there are many valuable studies that, for practical reasons, do not achieve ideal levels of power and precision. Thus, less-than-ideal power will not necessarily disqualify a paper from consideration at the journal (though, depending on the other strengths and weakness of the study, it could). Indeed, we would prefer that authors be honest about statistical power and allow reviewers and editors to consider this limitation in relation to other strengths that a study may have. Likewise, we hope all authors calibrate their interpretations with these issues in mind. In short, we believe that issues of statistical power and precision are critical for interpreting the conclusions that are drawn from a study, and thus, should be discussed openly as part of the review process.

\section{Data, Analytic Methods (Code), and Research Materials Transparency}

The editors of the Journal of Research in Personality believe that the data, methods used in the analysis, and materials used to conduct the research should be clearly and precisely documented and available to any researcher for purposes of reproducing the results, critically evaluating the conclusions of a report, or replicating the procedure. However, we also acknowledge that there are some situations where data and material sharing is not possible, either due to identifiable data, the use of proprietary data or instruments, or other similar circumstances. Accordingly, the policy of the Journal of Research in Personality is to require authors to make their data, materials, and code available to other researchers in a trusted digital repository, unless there is a reason why this is not possible. If data and code cannot be shared, then such reasons must be provided at the time of submission and indicated in an acknowledgment or footnote. Badges for open data and open materials will be provided for those papers where these resources are made public.

- Authors reusing data available from public repositories must provide program code, scripts for statistical packages, and other documentation sufficient to allow an informed researcher to precisely reproduce all published results.

- Authors using original data must:

- make the data available at a trusted digital repository (Note: If all data required to reproduce the reported analyses appears in the article text, tables, and figures then it does not also need to be posted to a repository.)

- include all variables, treatment conditions, and observations described in the manuscript.

- provide a full account of the procedures used to collect, preprocess, clean, or generate the data.

- provide program code, scripts, codebooks, and other documentation sufficient to precisely reproduce all published results.

- provide research materials and description of procedures necessary to conduct an independent replication of the research.

- In some cases, despite authors best efforts, some or all data or materials cannot be shared for legal or ethical reasons. In such cases, authors must inform the editors at the time of submission. This will be taken into account during the review process. Authors are encouraged to anticipate data and material sharing at the beginning of their projects to provide for these circumstances. It is understood that 
in some cases access will be provided under restrictions to protect confidential or proprietary information. Editors may grant exceptions to data and material access requirements provided authors:

- explain the restrictions on the dataset or materials and how they preclude public access.

- provide a public description of the steps others should follow to request access to the data or materials.

- provide software and other documentation that will precisely reproduce all published results.

- provide access to all data and materials for which the constraints do not apply.

- Data, program code, research materials, and other documentation of the research process should be made available through a trusted digital repository. Trusted repositories adhere to policies that make data discoverable, accessible, usable, and preserved for the long term. Trusted repositories also assign unique and persistent identifiers. For example these services are offered by partners in the Data Preservation Alliance for the Social Sciences (Data-PASS) and most institutional repositories. Authormaintained websites are not compliant with this requirement.

- Dissemination of these materials may be delayed until publication. Under exceptional circumstances, editors may grant an embargo of the public release of data for at most one year after publication.

- Articles accepted for publication will not be assigned a publication date until the above conditions have been met. Authors are responsible for ensuring that their articles continue to meet these conditions. Failure to do so may lead to an editorial expression of concern or retraction of the article.

The editors recognize that preparing materials to be shared and documenting datasets and materials requires effort. However, these efforts can also benefit authors, as the increased attention to detail often makes it easier to respond to issues raised in the review process and provides additional incentives for accurate reporting. We anticipate that these changes will reduce the need to publish corrections. Effort invested in archiving data and materials also helps to make ones own data more valuable for future meta-analyses, re-analyses, and other such efforts, which can increase the citation rate of a paper. Archiving can also make it more efficient to design future studies based on a well-documented initial study. Thus, these policies aimed at increasing transparency may end up benefiting authors as well as the field as a whole.

\section{Preregistration of Studies}

The policy of the Journal of Research in Personality is to publish papers where authors indicate whether the conducted research was preregistered with an analysis plan in an independent, institutional registry (e.g., http://aspredicted.org, http://openscienceframework.org). Preregistration of studies involves registering the study design, variables, as well as a thorough description of treatment conditions prior to conducting the research. Including an analysis plan involves specification of sequence of analyses or the statistical model that will be reported as well as detailing anticipated plans for excluding participants and detecting outliers. Given the importance of measurement to personality psychology, a preregistration plan for JRP may often require somewhat detailed descriptions of how measures will be scored and whether data transformations will be used. A link to the preregistration in an institutional registry must be made available to the journal prior to publication. The journal, or an entity acting on behalf of the journal, will verify that preregistration adheres to the specifications for preregistration and then provide certification of the preregistration in the article. Badges will be awarded for those studies that include a verifiable preregistration.

- Authors must, in acknowledgments or the first footnote, indicate if they did or did not preregister the research with or without an analysis plan in an independent, institutional registry.

- If an author did preregister the research, the author must

- confirm that the study was registered prior to conducting the research with links to the time-stamped preregistration(s) at the institutional registry, and that the preregistration adheres to the disclosure requirements of the institutional registry or those required for the preregistered badge with analysis plans maintained by the Center for Open Science.

- report all preregistered analyses in the text, or, if there were changes in the analysis plan following preregistration, those changes must be disclosed with explanation for the changes.

- clearly distinguish in text analyses that were preregistered from those that were not, such as having separate sections in the results for confirmatory and exploratory analyses.

The editors recognize that preregistration has sometimes proven controversial in past discussions. In some cases, these controversies were based on misunderstandings rather than serious problems with the technique. We reiterate that preregistration is not a requirement for publication in JRP and we recognize that not all types of research are easily amenable to preregistration. However, the editors believe there is value in promoting preregistration in personality psychology. To be sure, even secondary data analysts can register their analytic plans before analyses are conducted. Preregistration helps to clarify distinctions between confirmatory and exploratory research and may help reduce subtle and even relatively unconscious forms of p-hacking (Simmons et al., 2011) or walks down the garden of forking paths (Gelman \& Loken, 2013). Preregistration provides researchers with a record of their initial intentions so they have a clear reference point to judge how far they deviated 
from their original plans. Preregistration plans make it easier to develop a more realistic appraisal of how many alternative analyses were conducted so that authors can have a more realistic assessment of the strength of the evidence provided by their analyses. Moreover, there is often much to be gained by thinking carefully about complicated data analytic issues before data are collected and analyses are conducted.

\section{Registered Reports}

Although the TOP guidelines do not specify whether a journal should adopt the use of registered reports (where papers get an in principle acceptance based on the introduction and methods section; see Nosek \& Lakens 2014), the Journal of Research in Personality will make this option available for authors. We note that registered reports have been quite rare in personality psychology, perhaps because of a greater acceptance of descriptive or purely exploratory research where the informational value of the study is interesting regardless of the outcome of a particular statistical analysis. However, we also believe that it would benefit the field to think carefully about how registered reports could be used to promote better research practices in personality psychology. There are many cases where researchers might identify a method for addressing an interesting and important research question. It is quite valuable to then have that method independently and critically evaluated before it is used. This step saves authors from collecting data using a method that was not favorably evaluated by peers, and obviate pressures to simply find an effect because of all of the efforts that went into data collection. Moreover, registered reports can help reduce the file drawer problem because it creates a mechanism for publishing the results of designs that were initially deemed to be reasonable tests. Null results are informative when the design of a study was strong and compelling. In contrast, null results from poorly designed studies often have little informational value. Registered reports provide a mechanism for evaluating the strength of the method apart from whether or not a focal p-value was less than a particular alpha level.

Thus, going forward, the journal will consider such submissions. Authors who wish to use the registered report option should note this in their cover letter and should follow a twostage process (see the guide to authors for more details:

- On initial submission, authors should:

- Note in the cover letter that the manuscript is a Registered Report submission and confirm that the data do not exist, or that the outcomes have not been observed.

- include a full manuscript for the abstract, introduction, and methods without the results and discussion sections.

- disclose that the methods contain a complete analysis plan of what is to be included in the full article.
- If the submission passes initial review, then the authors will receive an in-principle acceptance prior to data collection or analysis of the outcomes. This in-principle acceptance means that the authors can proceed to the second stage of review.

- For the second stage of review, authors submit a complete manuscript. Reviewers assess the extent to which the authors followed the preregistered design and/or analysis plan, and evaluate non-outcome relevant criteria (e.g., manipulation checks) to confirm whether the research was an effective test of the research question.

\section{Replication}

The policy of the Journal of Research in Personality for at least the past four years (Lucas \& Donnellan, 2013) has been to encourage submission of replication studies, particularly of research published in this journal. In our previous policy changes, we actively encouraged replication studies and provided an abbreviated review option specifically for replications of studies that had been published in the journal within the previous five years. The idea behind the second part of this policy was that the recent papers published in the journal had already been vetted for importance and design, and thus, replication attempts only needed to be evaluated on the basis of whether they were high-quality replications. Replication attempts for studies that were not recently published in the journal would need to go through a standard review process, where the question and design were evaluated by experts.

As part of our adoption of the TOP guidelines, we are modifying this replication policy to incorporate results-blind review of all replication studies and to encourage the use of registered reports. When possible, replication studies are reviewed in two stages following the registered reports format described above. For replication studies where data have already been conducted, "results-blind" review will be used. The process is essentially the same as what is described above: authors should submit an abstract, introduction, and method section (including detailed analysis plan) for the first stage of review, followed by a secondstage review where results and discussion are provided.

\section{Additional Disclosures}

Finally, to increase openness, transparency, and reproducibility, we are asking authors to include additional disclosures in the manuscripts they submit. Specifically, regarding author contributions, authors should include a footnote explaining the contributions for which each author was responsible (e.g., study conceptualization, data collection, data preparation, data analysis, report writing). In addition, to adequately constrain conclusions, authors should discuss any limitations they see on the generalizability of their findings. For instance, if they think their finding will only hold among specific populations, in specific historical contexts, or with specific experimental materials, authors should discuss these limitations in their paper. As noted above, this will provide appropriate constraints on the 
conclusions that are drawn, but can also be useful information for other researchers who wish to replicate these results (see, Simons et al., 2017).

\section{Summary}

Openness and transparency are critical for science. The Journal of Research in Personality is committed to improving the incentives that authors have for conducting personality research in an open and transparent way. The choices we made in regard to the TOP guidelines were made with consideration both of their benefits and of any potential burden these changes place on authors and any side effects these changes may have in terms of the types of research that can be published here. Ultimately, the changes in policy that we have adopted simply change the default behavior (while still allowing for exceptions) and provide new options for submitting and evaluating manuscripts (such as the registered reports). We hope that these changes show our commitment to publishing strong and creative research on personality that is also transparent, open for evaluation, and ideally robust.

\section{References}

Cohen, J., Cohen, P., West, S. G., \& Aiken, L. S. (2003). Applied Multiple Regression/Correlation Analysis for the Behavioral Sciences. Mahwah, NJ: Lawrence Erlbaum Associates, Inc.

Gelman, A., \& Loken, E. (2013). The garden of forking paths: Why multiple comparisons can be a problem, even when there is no "fishing expedition" or "p-hacking" and the research hypothesis was posited ahead of time. Downloaded from: http://www.stat.columbia.edu/ /gelman/research/unpublished/p_hacking.pdf.

Gignac, G. E., \& Szodorai, E. T. (2016). Effect size guidelines for individual differences researchers. Personality and Individual Differences, 102, 74-78.

Kidwell, M. C., Lazarević, L. B., Baranski, E., Hardwicke, T. E., Piechowski, S., Falkenberg, L.-S., Kennett, C., Slowik, A., Sonnleitner, C., Hess-Holden, C., Errington, T. M., Fiedler, S., \& Nosek, B. A. (2016). Badges to Acknowledge Open Practices: A Simple, Low-Cost, Effective Method for Increasing Transparency. PLOS Biology, 14, e1002456.

Lucas, R. E., \& Donnellan, M. B. (2013). Improving the replicability and reproducibility of research published in the Journal of Research in Personality. Journal of Research in Personality, 4, 453-454.

Maxwell, S. E., Kelley, K., \& Rausch, J. R. (2008). Sample Size Planning for Statistical Power and Accuracy in Parameter Estimation. Annual Review of Psychology, 59, 537-563.

Nosek, B. A., Alter, G., Banks, G. C., Borsboom, D., Bowman, S. D., Breckler, S. J., Buck, S., Chambers, C. D., Chin, G., Christensen, G., Contestabile, M., Dafoe, A., Eich, E., Freese, J., Glennerster, R., Goroff, D., Green, D. P., Hesse, B., Humphreys, M., Ishiyama, J., Karlan, D., Kraut, A., Lupia, A., Mabry, P., Madon, T., Malhotra, N., Mayo-Wilson, E., McNutt, M., Miguel, E., Paluck, E. L., Simonsohn, U., Soderberg, C., Spellman, B. A., Turitto, J., VandenBos, G., Vazire, S., Wagenmakers, E. J., Wilson, R., \& Yarkoni, T. (2015). Promoting an open research culture. Science, 348, 1422-1425.

Nosek, B. A., \& Lakens, D. (2014). Registered Reports. Social Psychology, $45,137-141$.

Richard, F. D., Bond, C. F., \& Stokes-Zoota, J. J. (2003). One Hundred Years of Social Psychology Quantitatively Described. Review of General Psychology, 7, 331-363.

Simmons, J., Nelson, L., \& Simonsohn, U. (2011). False-Positive Psychology Undisclosed Flexibility in Data Collection and Analysis Allows Presenting Anything as Significant. Psychological Science, 22, 1359-1366.

Simons, D. J., Shoda, Y., \& Lindsay, D. S. (2017). Constraints on Generality (COG): A Proposed Addition to All Empirical Papers. Manuscript submitted for publication.

Simonsohn, U. (2014). No-way Interactions. Http://datacolada.org/17. 